\title{
New expectations from the well-known medicinal properties of Arctium lappa
}

\author{
C. Miele $\cdot$ F. Beguinot
}

Received: 11 November 2011 / Accepted: 5 January 2012/Published online: 23 February 2012

(C) Springer-Verlag 2012

\begin{abstract}
AMP-activated protein kinase (AMPK) serves as a major regulator of energy homeostasis and is activated by different glucose-lowering agents. Indeed, AMPK has been identified as an attractive target for the development of innovative molecules to treat type 2 diabetes. In this issue of Diabetologia (doi: 10.1007/s00125-011-2366-3), Huang and co-workers report that arctigenin activates muscle uptake of glucose and inhibits hepatocyte gluconeogenesis and lipogenesis by reducing mitochondrial respiration and inducing AMPK activity. Importantly, it is reported that arctigenin improves glucose and lipid metabolism in $o b / o b$ mice. Based on this evidence, Huang and co-workers suggest that arctigenin may represent a valuable lead compound for developing innovative glucose-lowering molecules. While these findings are not entirely novel and mechanistic investigations are needed, the results strongly support the concept that arctigenin deserves to be further considered because of its several potentially beneficial in vivo effects. In particular, the authors conclude that further mechanistic studies on arctigenin might provide novel insight and opportunities for selective modulation of subcutaneous and visceral fat mass.
\end{abstract}

Keywords AMPK $\cdot$ Arctigenin $\cdot$ Type 2 diabetes

C. Miele $\cdot$ F. Beguinot $(\square)$

Dipartimento di Biologia e Patologia Cellulare e Molecolare, Università di Napoli Federico II,

Via S. Pansini, 5,

80131 Naples, Italy

e-mail: beguino@unina.it

C. Miele $\cdot$ F. Beguinot

Istituto di Endocrinologia ed Oncologia Sperimentale del CNR, Naples, Italy

\begin{abstract}
Abbreviations
AMPK AMP-activated protein kinase

CaMKK $\mathrm{Ca}^{2+} /$ calmodulin-dependent protein kinase kinase LKB1 Liver kinase B1
\end{abstract}

Type 2 diabetes is a leading cause of morbidity and mortality all over the world. Successful management of type 2 diabetes requires strict control of glycaemia and other risk factors to prevent the progression of the disease. Despite the availability of a number of oral glucose-lowering drugs and convenient insulin preparations, the majority of patients fail to maintain tight glycaemic control over time, raising their risk of developing micro- and macrovascular complications. [1]. A key reason for this therapeutic gap may be that current therapies and strategies do not adequately address all of the underlying causes of the disease. If this is the case, it is pivotal to develop further drugs that restore normoglycaemia by targeting specific pathogenic defects $[2,3]$.

Type 2 diabetes is accompanied by abnormalities affecting an impressive number of distinct signalling pathways $[4,5]$. At present, the discovery of novel molecules targetting these pathways represents a powerful approach for the development of glucose-lowering drug lead compounds. Natural products offer a major resource of biologically active molecules for screening $[4,6]$, and active components from plants and marine organisms featuring glucoselowering activity are constantly being identified. Indeed, a large number of natural small molecules have been reported to modulate key signalling pathways involved in type 2 diabetes [4]. These pathways are implicated in insulin signal transduction, insulin secretion, glucose metabolism, peroxisome proliferator-activated receptor regulation, endoplasmic reticulum stress and inflammation and chromatin modification. 
AMP-activated protein kinase (AMPK) is a highly conserved serine/threonine protein kinase that serves as a major regulator of energy homeostasis both at the cellular and whole body level [7, 8]. AMPK coordinates a complex network of signalling pathways, ultimately balancing nutrient supply and energy requirements (Fig. 1). In animal models of insulin resistance and type 2 diabetes, pharmacological activation of AMPK has been shown to improve blood glucose homeostasis, lipid profile and blood pressure [4, 9] and mimics the beneficial effects of physical exercise and energy restriction on these variables. In line with this, AMPK is activated by major glucose-lowering molecules, including the biguanide metformin and the thiazolidinediones [10], and by the insulinsensitising adipokine adiponectin [10]. These findings have provided a rationale for drug discovery projects aimed at identifying novel AMPK-targeting agents for treating type 2 diabetes and the metabolic syndrome. Targeting AMPK with innovative molecules might present unanticipated difficulties, however, because of unwanted tumour-promoting effects and heart problems [10]. In addition, AMPK activation at the hypothalamic level may cause further undesired consequences, including increased body weight [10]. Thus, development of innovative medicines targeting AMPK must take a number of potential problems into account.

In this issue of Diabetologia, Huang et al. [11] report that the natural dibenzylbutyrolactone-type lignanolide arctigenin, a molecule extracted from the traditional herb Arctium lappa L. (Compositae) that has previously been shown to feature anti-tumour and anti-inflammatory effects [12, 13], increases AMPK phosphorylation. In addition, they show that arctigenin stimulates glucose uptake in cultured L6 skeletal muscle

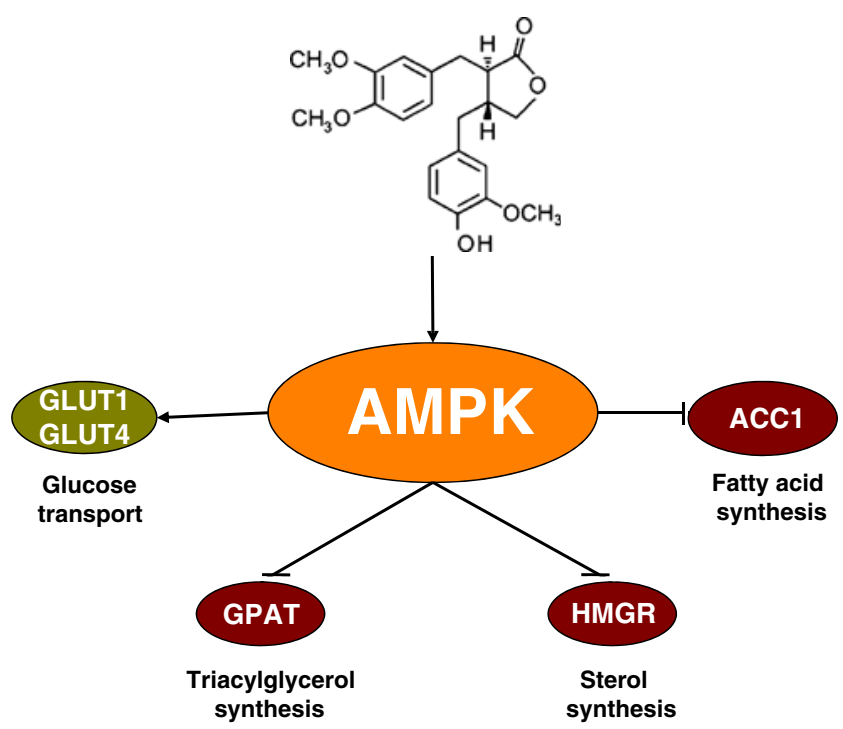

Fig. 1 AMPK-mediated effects of arctigenin on skeletal muscle and liver. Arctigenin-activated AMPK induces glucose uptake $(\rightarrow)$ and blocks $(\dashv)$ fatty acid, sterol and triacylglycerol synthesis. ACC1, acetyl-CoA carboxylase 1; GPAT, glycerol-3-phosphate acyltransferase; HMGR, 3-hydroxy-3-methylglutaryl-CoA reductase cells and isolated muscles, while inhibiting gluconeogenesis and lipid synthesis in primary hepatocytes [11]. Interestingly, all of these effects were blocked by AMPK $\alpha 1 / \alpha 2$ silencing or pharmacological inhibition of AMPK, whereas pharmacological inhibition of phosphoinositide-3 kinase had no effect. Arctigenin also inhibits the respiration of L6 myotubes and of isolated mitochondria through a specific effect on respiratory complex I. Finally, the authors show that chronic administration of arctigenin lowers blood glucose levels and improves lipid metabolism in $o b / o b$ mice, while acute oral administration is effective in inhibiting gluconeogenesis even in wild-type mice. Based on this evidence, they conclude that arctigenin activates AMPK by inhibiting respiratory complex I and improves metabolic derangement in the $o b / o b$ mouse model. The authors suggest that this molecule is of value as a lead compound in drug discovery strategies looking for innovative treatments for type 2 diabetes.

Tang et al., working at the same institution as Huang, recently published an elegant paper showing that arctigenin enhances treadmill endurance in sedentary mice and activates $\mathrm{AMPK}$ in $\mathrm{C} 2 \mathrm{C} 12$ cultured muscle cells [14]. These authors also concluded that arctigenin might be used as a lead compound for diabetes drug discovery. Thus, the hypothesis that arctigenin research may lead to the identification of agents mimicking the beneficial effects of exercise on metabolic disorders is not a new concept. However, the report by Huang et al. [11] is the first to show a positive effect of arctigenin administration on glucose tolerance and lipid profile in mice. This advance is limited by the lack of in-depth investigation of the mechanisms responsible for the effects of arctigenin. Whether this agent affects in vivo glucose disposal, hepatic gluconeogenesis and insulin secretion and action remains to be conclusively demonstrated. The relative contribution of each of these potential effects also remains to be explored.

Interestingly, it has been reported that arctigenin enhances AMPK phosphorylation and activity through $\mathrm{Ca}^{2+} /$ calmodulin-dependent protein kinase kinase (CaMKK)- and liver kinase B1 (LKB1)-dependent pathways in $\mathrm{C} 2 \mathrm{C} 12$ and $\mathrm{H} 9 \mathrm{C} 2$ cells [14]. However, Huang and co-workers [11] found that this mechanism does not occur in the L6 muscle cell line. Instead, arctigenin-induced activation of AMPK occurs indirectly and is not dependent upon LKB1, CaMKK or the nitric oxide synthase cascades. Whether arctigenin exerts differential effects in different muscle cell lines and, maybe more importantly, whether and how its biological action exhibits tissue specificity remain unknown and deserve further attention.

In their paper, Huang et al. report another piece of information that is both puzzling and potentially of interest. In the mouse model, chronic treatment with arctigenin caused a significant reduction in subcutaneous fat with no change in either visceral fat or body weight. The reasons for the differential effect of arctigenin on subcutaneous vs visceral fat were 
not addressed and deserve to be explored in the future, as they might provide novel insight and opportunities for selective modulation of subcutaneous and visceral fat mass in mammals. Why such a significant reduction in subcutaneous fat occurs with no change in total body mass in the arctigenintreated mice also remains a mystery, as does the issue of which tissue(s) compensates in mass for the loss of subcutaneous fat.

Cancer is being increasingly recognised as a major problem in diabetes $[15,16]$. Indeed, many forms of cancer show an increased prevalence among diabetic individuals $[15,16]$ and the clinical outcome of patients with diabetes is often worse $[15,16]$. The mechanisms responsible for this association are ultimately unknown and may include molecular abnormalities common to cancer and diabetes. Arctigenin has been identified as an inhibitor of protein kinase B/Akt activation [17]. Recent reports indicate that this feature is key to the known anti-tumour activity of arctigenin $[18,19]$. Interestingly, Huang et al. report that chronic administration of arctigenin results in a significant increase in insulinstimulated activation of Akt [11]. Clearly, the effect of arctigenin on Akt deserves to be further investigated as it might shed light onto the molecular mechanisms that determine the impact of diabetes on tumour progression.

In summary, the glucose-lowering properties of Arctium lappa may reveal much about the mechanisms underlying glucose tolerance abnormalities. Whether this information will contribute to the search for innovative glucose-lowering drugs remains unclear.

Funding This work was supported by the European Foundation for the Study of Diabetes (EFSD), the European Community's PREPOBEDIA (201681), grants from the Associazione Italiana per la Ricerca sul Cancro (AIRC), from the Ministero dell'Università e della Ricerca Scientifica (PRIN programmes to C. Miele and F. Beguinot, and the MERIT initiative, FIRB program: RBNE08NKH7). The financial contribution of Telethon Italy is gratefully acknowledged.

Duality of interest The authors declare that there is no duality of interest associated with this manuscript.

Contribution statement Both CM and FB analysed and interpreted the data described in this commentary and contributed to the drafting and revising of the article. Both authors approved the final version of the article.

\section{References}

1. Cohen A, Horton ES (2007) Progress in the treatment of type 2 diabetes: new pharmacologic approaches to improve glycemic control. Curr Med Res Opin 23:905-917

2. Polakof S (2010) Diabetes therapy: novel patents targeting the glucose-induced insulin secretion. Recent Pat DNA Gene Seq $4: 1-9$

3. Tahrani AA (2011) Management of type 2 diabetes: new and future developments in treatment. Lancet 378:182-197

4. Liu Q, Chen L, Hu L et al (2010) Small molecules from natural sources, targeting signalling pathways in diabetes. Biochim Biophys Acta 1799:854-865

5. Nolan CJ, Damm P, Prentki M (2011) Type 2 diabetes across generations: from pathophysiology to prevention and management. Lancet 368:169-181

6. Newmann DJ, Cragg GM (2007) Natural products as sources of new drugs over the last 25 years. J Nat Prod 70:461-477

7. Hardie DG (2011) AMP-activated protein kinase: an energy sensor that regulates all aspects of cell function. Genes Dev 25:1895-1908

8. Carling D, Mayer FV, Sanders MJ, Gamblin SJ (2011) AMP-activated protein kinase: nature's energy sensor. Nat Chem Biol 7:512-518

9. Cool B, Zinker B, Chiou W et al (2006) Identification and characterization of small molecule AMPK activator that treats key components of type 2 diabetes and the metabolic syndrome. Cell Metab 3:403-416

10. Yun H, Ha J (2011) AMP-activated protein kinase modulators: a patent review (2006-2010). Expert Opin Ther Pat 21:983-1005

11. Huang SL, Yu R-T, Gong J et al (2011) Arctigenin, a natural compound, activates AMP-activated protein kinase via inhibition of mitochondria complex I and ameliorates metabolic disorders in ob/ob mice. Diabetologia. doi:10.1007/s00125-011-2366-3

12. Yao X, Zhu F, Zhao Z, Liu C, Luo L, Yin Z (2011) Arctigenin enhances chemosensitivity of cancer cells to cisplatin through inhibition of the STAT3 signaling pathway. J Cell Biochem 112:2837-2849

13. Lee JY, Kim CJ (2010) Arctigenin, a phenylpropanoid dibenzylbutyrolactone lignan, inhibits type I-IV allergic inflammation and pro-inflammatory enzymes. Arch Pharm Res 33:947-957

14. Tang X, Zhuang J, Chen J et al (2011) Arctigenin efficiently enhanced sedentary mice treadmill endurance. PLoS One 6:1-13

15. Smith U, Gale EAM (2009) Does diabetes therapy influence the risk of cancer. Diabetologia 52:1699-1708

16. Giovannucci E, Harlan D, Archer MC (2010) Diabetes and cancer: a consensus report. Diabetes Care 33:1674-1685

17. Kim Y, Hollenbaugh JA, Kim DH, Kim B (2011) Novel PI3K/Akt inhibitors screened by the cytoprotective function of human immunodeficiency virus type 1 Tat. PLoS One 6:e21781

18. Awale S, Lu J, Kalauni SK et al (2011) Identification of arctigenin as an antitumor agent having the ability to eliminate the tolerance of cancer cells to nutrient starvation. Cancer Res 66:1751-1757

19. Gui-Rong C, Li-Ping C, De-Qiang D et al (2012) Synthesis of $(-)$-arctigenin derivatives and their anticancer activity. Nat Prod Res 26:177-181 